\title{
A SUM IN TERMS OF THE DIRICHLET BETA FUNCTION
}

\author{
SUMIT KUMAR JHA
}

ABSTRACT. In this note, we derive the following sum

$$
\beta(s)=\sum_{n=1}^{\infty} \frac{(-1)^{n}}{(2 n+1)^{s}}=-\sum_{1 \leq i_{1} \leq i_{2} \leq \cdots \leq i_{s}} \frac{2_{s}^{i_{s}-1}}{i_{s}\left(\begin{array}{l}
2 i_{s} \\
i_{s}
\end{array}\right)} \cdot \frac{1}{\left(1+2 i_{1}\right)\left(1+2 i_{2}\right) \cdots\left(1+2 i_{s}\right)},
$$

where $s$ is any natural number, and the summation is over all $s$-tuples $\left(i_{1}, i_{2}, \cdots, i_{s}\right)$ of natural numbers satisfying $1 \leq i_{1} \leq i_{2} \leq \cdots \leq i_{s}$ for fixed $s$.

\section{MAin RESUlT}

Theorem 1. Let s be any natural number. Then we have

$$
\beta(s)=\sum_{n=1}^{\infty} \frac{(-1)^{n}}{(2 n+1)^{s}}=-\sum_{1 \leq i_{1} \leq i_{2} \leq \cdots \leq i_{s}} \frac{2^{i_{s}-1}}{i_{s}\left(\begin{array}{c}
2 i_{s} \\
i_{s}
\end{array}\right)} \cdot \frac{1}{\left(1+2 i_{1}\right)\left(1+2 i_{2}\right) \cdots\left(1+2 i_{s}\right)} .
$$

Proof. To derive equation (1), we use the following result from [1]

$$
\begin{array}{r}
\mathbf{L i}_{s}^{\alpha}(w)=\sum_{n=1}^{\infty} \frac{w^{n}}{(\alpha+n)^{s}} \\
=-\sum_{1 \leq i_{1} \leq \cdots \leq i_{s}} \frac{\left(i_{s}-1\right) !}{(\alpha+1)(\alpha+2) \cdots\left(\alpha+i_{s}\right)} \cdot \frac{1}{\left(\alpha+i_{1}\right)\left(\alpha+i_{2}\right) \cdots\left(\alpha+i_{s-1}\right)} \cdot\left(\frac{-w}{1-w}\right)^{i_{s}}
\end{array}
$$

Letting $\alpha=1 / 2$ snd $w=-1$ in the above we get (1) at once.

\section{SPECiAL CASES}

Letting $s=1$ in equation (1) we get

$$
\frac{\pi}{4}=1-\sum_{i=1}^{\infty} \frac{2^{i-1}}{i\left(\begin{array}{c}
2 i \\
i
\end{array}\right)} \cdot \frac{1}{(1+2 i)}
$$

Remark 1. Some other such type of sums have also been obtained in [2].

2010 Mathematics Subject Classification. 40B05, $11 \mathrm{M06.}$

Key words and phrases. Dirichlet beta function. 


\section{REFERENCES}

1. Emery. M, On a multiple harmonic power series, Preprint; available at http://arxiv.org/abs/math/0411267v2 (2004)

2. Jha, Sumit K. 2019. Some Sums in Terms of the Riemann Zeta Function, OSF Preprints. December 31. doi:10.31219/osf.io/47hm5

INTERNATIONAL INSTITUTE OF INFORMATION TECHNOLOGY, HYDERABAD, INDIA

E-mail address: kumarjha.sumit@research.iiit.ac.in 